\title{
Expressivity in Interaction: a Framework for Design
}

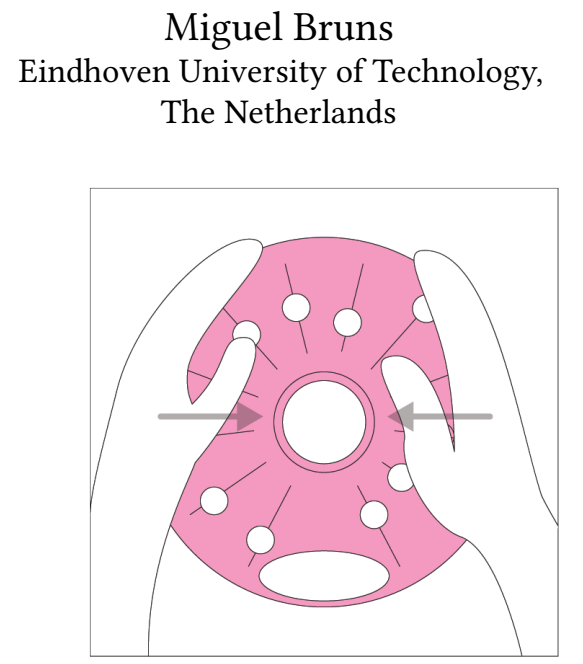

\author{
Stijn Ossevoort \\ Eindhoven University of Technology, \\ The Netherlands
}
Marianne Graves Petersen
Aarhus University, Denmark

Figure 1: Research artefacts illustrating different perspectives on expressivity in HCI. Left the alarm clock by Wensveen et al. [76] emphasizing human emotional expressivity. Middle PLANKS by Vallgårda [70] emphasizing artefact expressivity. Right illustrates expressivity in interaction through an interactive lamp, AEI by Ross [61].

\begin{abstract}
Expressivity is frequently recurring as a term in HCI, but it is often approached from different perspectives. Affective computing prompted research into emotional expressivity, and with technology becoming more ubiquitous and tangible, the opportunities for expressive behaviors towards systems as well as the expressivity of systems increases. By analyzing exemplar research-through-design cases and a literature survey on the use of expressivity in interaction, we discuss how different perspectives and concepts contribute to understand expressivity in interaction. We integrate these perspectives and make them operational for interaction design by creating a framework including design considerations such as freedom of interaction, action-perception loops, multimodality, subtlety, ambiguity, skill development and temporal form. The framework is a result of a mixed-method approach including a review of existing definitions and scholarly artefacts, and a systematic literature review to identify design cases including an analysis of these design cases. We finally illustrate how the framework has been used to inform the design of a shape-changing soft-robotic interface. As a result, we contribute an integrated framework on how to design for expressivity in interaction.
\end{abstract}

This work is licensed under a Creative Commons Attribution International 4.0 License.

CHI '21, May 08-13, 2021, Yokohama, Japan

(C) 2021 Copyright held by the owner/author(s)

ACM ISBN 978-1-4503-8096-6/21/05.

https://doi.org/10.1145/3411764.3445231

\section{CCS CONCEPTS}

- Human-centered computing; • Interaction design; • Interaction design theory, concepts and paradigms.

\section{KEYWORDS}

Expressivity in Interaction, Research-through-Design, Framework, Design Case

\section{ACM Reference Format:}

Miguel Bruns, Stijn Ossevoort, and Marianne Graves Petersen. 2021. Expressivity in Interaction: a Framework for Design. In CHI Conference on Human Factors in Computing Systems (CHI '21), May 08-13, 2021, Yokohama, fapan. ACM, New York, NY, USA, 13 pages. https://doi.org/10.1145/3411764.3445231

\section{INTRODUCTION}

As interactive technology is increasingly becoming part of our lifeworld, several voices have raised the need for broadening our conception of how we understand and design human-computer interaction [27, 67]. Our work is particularly triggered by technological trends such as artificial intelligence, the miniaturization of actuators, emerging materials supporting a materiality approach to HCI [26], and shape-changing interfaces [54], which illustrate how technology increasingly becomes interwoven in our everyday artefacts and environments. As a response to the ever more pervasive use of technology in everyday life, there seems to be an increasing focus around expressivity in HCI. By studying more specifically how the term expressivity is adopted throughout the HCI literature, we see that the notion of expressivity embraces rather different perceptions of the term. Expressivity has been considered to indicate that actions towards products or systems can carry affective and emotional content $[9,20]$; it has been seen as relating to the visceral capacity of the human body [10] and to bodily movements such as touch, posture and gesture and their unique qualities [44]. Beyond 


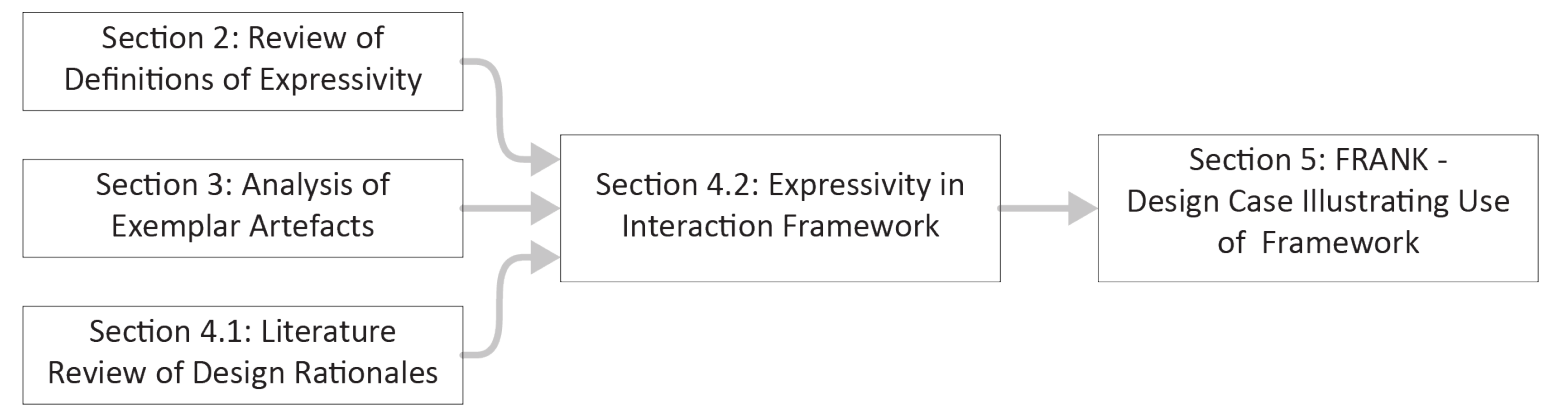

Figure 2: Outline of the paper

human expressiveness it is also used to emphasize the diversity of qualities within modalities or domains such as music [17, 73], as well as the interaction between multiple modalities [2]. Furthermore, expressivity has been used to articulate the way objects may arouse a feeling or emotion [29] or the extent to which they allow users to express themselves emotionally $[64,77]$. Expressive interaction is seen as supporting the development of a relationship or a better fit between user and product [22] and some see expressive communication as being opposed to goal-oriented and informative communication [66]. Expressive interaction has been related to the wider agenda of broadening the way we understand how interactive technology is part of our life worlds [27, 67].

As can be seen in the variety of explanations above, there is a cacophony of voices talking about the same or closely related terms (expression, expressive behavior, expressiveness, expressivity), but the problem with expressivity in $\mathrm{HCI}$ is that, while many researchers emphasize that interaction should be expressive, there is little agreement on what the term denotes. The field contains a plethora of perspectives on expressivity comprising different fundamental assumptions about the role of technology, the role of expressivity and related criteria for good interaction. Asking the provocative question "What is interaction?" Hornbæk and Oulusvirta [32] have convincingly demonstrated that we need further development of the core concepts in the field of HCI. Thereupon, we propose expressivity as such a core concept in HCI. We motivate the need for a more elaborate vocabulary around expressivity and the need to support designers in designing for expressivity in interaction. Therefore, this paper aims at bringing expressivity to the forefront and to establish it as a first-class citizen in the field of $\mathrm{HCI}$ [32].

In addition, we would like to point out that research into expressivity in HCI stems from different schools of thought and disciplines, which requires a multi-method approach. Therefore we chose several angles, which compose the underlying structure of this paper (see figure 2). First, we review existing definitions of expressivity in $\mathrm{HCI}$; second, we analyze a set of scholarly research-through-design artefacts (see e.g. figure 1), emphasizing expressivity in their design rationales; and we investigate their underlying perspectives and outline how these artefacts articulate interaction qualities. Third, we investigate expressivity in interaction by conducting a literature review identifying design cases which explicitly refer to expressivity in their design rationale. For this phase, we extracted a set of 251 papers from the ACM conferences CHI (Human Factors in Computing Systems), TEI (Tangible Embedded and Embodied Interaction) and DIS (Designing Interactive Systems) in the period from 2016 to 2020 dealing with expressivity, interaction and design, and annotated a subset of 29 papers that describe design cases and guidelines regarding expressivity in interaction. Fourth, based on our analysis we developed a framework to support designers in their practice. Fifth, we illustrate how the framework has been used by interaction design students to inform the design of a shape-changing soft-robotic interface named FRANK. Finally, we discuss a possible agenda for future HCI research into expressivity in interaction.

\section{DEFINITIONS OF EXPRESSIVITY}

It is striking that papers addressing expressivity in HCI contain very few definitions of the term. A small set of authors describe the term expressivity, and in some cases, they make the connection to expressive interaction. Wilkinson et al. describe expressivity as bodily actions that convey "rich and complex intent" and relate to expressive interaction as a temporal account of different interaction opportunities [78]. Similarly, Piana et al. emphasize that expressivity relates to different human body movement qualities [52] and Benyon et al. describe how expressive interaction is influenced by bodily actions such as speech, touch and gestures [2]. Camurri et al. also refer to expressive body movements but indicate that these movements are used to convey aspects related to emotions [9]. This connects to Hook et al. who use a definition from the Oxford Dictionary for expression: "the action of making known one's thoughts or feelings" [29]. They emphasize that expressive interaction revolves around a dialogue between the human and the system. This is exemplified by Wensveen and Overbeeke who suggest that the expressiveness of how users interact with a product (reflected through actions towards a system) correlates with the emotional state of the person [76]. Similarly, Dalsgaard et al. define expressivity as "the way and degrees to which people can convey thoughts or feelings" when interacting with a system [12].

Not only do Dalsgaard et al. address the ability to express oneself towards a system using different types of movements, they also discuss the extent to which the system responds to these movements through multiple modes of expression, such as text, audio or video [13]. Reeves et al. describe how expressive interfaces respond to actions by revealing or amplifying certain effects expressed by the system and how systems intend to engage and entertain spectators [56]. Forlizzi and Battarbee discuss how expressive interfaces support in developing a relationship between a user and a system by changing, modifying and personalizing the system. This effort 


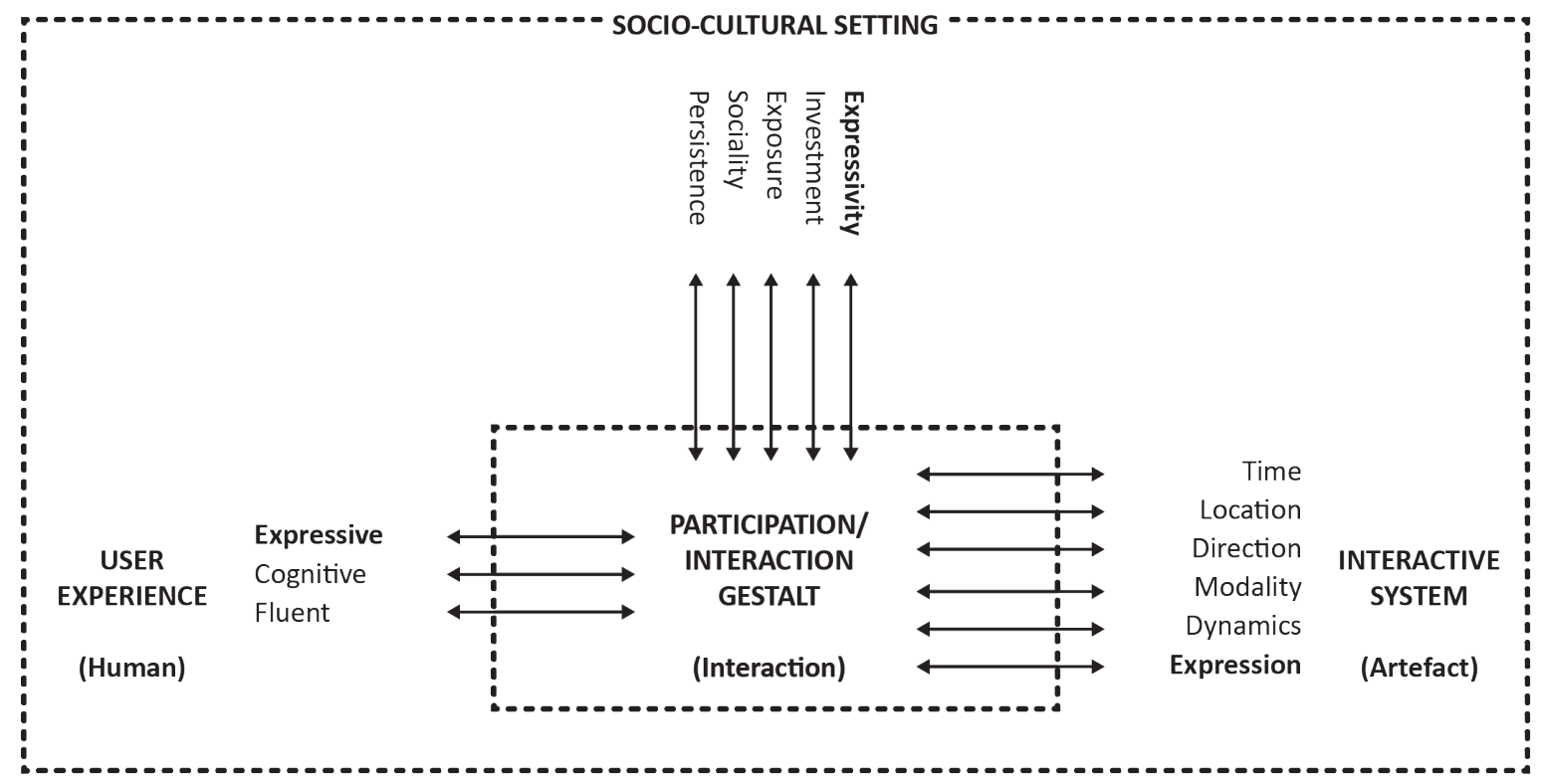

Figure 3: Considerations of human expressivity in user-product interactions in the framework of User Experience [22] (left); of system expressivity in the six aspects of coupling action and function in the Interaction Frogger Framework [75] (right); and how expressivity relates to the socio-cultural context as one of the five participatory qualities in the Participation Gestalt Framework [12] (figure inspired by Dalsgaard et al. [12]).

supports the development of a better fit [22]. The design for a better fit is more extensively described by Ross and Keyson who present a selection of design principles for expressive tangible interfaces [60]. They discuss that for an interface to be expressive designers must consider the context of interaction and start from the experience. To map the human expressivity to the action possibilities of the artefact, designers should have a clear understanding of potential types of action, develop a model for translating action, freedom of action (if necessary constrained by physical action possibilities) and a model for translating the expressive behavior of the device, which can be achieved in qualities such as physical form, temporal form or materiality. Finally, they emphasize how designers should consider the subjective nature of expression.

As can be seen, many definitions of expressivity center around human ability to express themselves in terms of movement, actions, intent, and emotions when interacting with a system $[2,9,52,76,78]$. But Hook et al. also discuss the dialogical nature of the interaction [29] and Dalsgaard et al. [12] emphasize that expressivity is also a matter of how the system responds through e.g. multiple modalities. We find these different perspectives representative for the three core elements of expressivity and interaction, namely, emphasizing the human, artefact and interaction. Figure 3 illustrates how the three perspectives connect. In their Framework of User Experience Forlizzi and Batterbee identify expressivity as one of the three userproduct interactions. They indicate that expressive interactions support the development of a relationship between a user and a system [22]. In their Frogger Framework, which supports designers in analyzing and synthesizing the feedback and feedforward of interactive systems, Wensveen et al. consider expressivity as one of the six aspects that need to be coupled by means of feedback and feedforward between system functionality and users' actions. They refer to expression as a system's reaction, with a diversity of dynamic qualities, reflecting the expression of a user's action [75]. Finally, the two perspectives (human and system) are incorporated into a model illustrating the Participation Gestalt Framework that combines the user experience and interactive systems, but also includes the socio-cultural setting. Dalsgaard et al. identify five continua of participatory qualities, including expressivity, that compose the Participation Gestalt. They define expressivity as the way in which people can convey thoughts or feelings towards a system using multiple, multimodal and nuanced approaches, depending on their competences [12]. In the following, we elaborate upon the three perspectives and their socio-cultural context, before we study a selection of scholarly research-through-design artefacts.

\section{PERSPECTIVES EMBEDDED IN EXEMPLAR RESEARCH-THROUGH-DESIGN ARTEFACTS}

Despite the lack of common definitions, expressivity has been a prominent term in the design rationales for various researchthrough-design projects. In fact, one of the initial motivations for this paper was our encounter with a series of research artefacts seeking to push the roles and nature of computational artefacts in our life-worlds and providing expressivity as part of the design rationale [26, 48]. Acknowledging the nature of research-throughdesign implies that we can learn from studying these artefacts and their rationales [16], the perspectives they embody, the design choices made, the underlying values, and in this way understand more of the implicit theories embedded in the artefacts. In the same 
vein, Carroll and Kellogg emphasize the role of $\mathrm{HCI}$ artefacts as theory nexus and have pointed out that in HCI artefacts often precede the theory [11]. We suggest that this is very much the situation for expressivity in interaction. Accordingly, we move on to study the perspectives and rationales inherent in prominent research artefacts related to expressivity.

For our analysis, we build on the Perspectives Framework by Ross [59], which distinguishes between the human behavior, the artefact behavior, and their interaction and provides four perspectives of engagement, Sensory-Motor Activity, Dynamic Form, Social Activity, and Value. We structure our analysis in line with these perspectives: one group of artefacts focuses on how to support and enable human expressivity; another group of artefacts explores new opportunities for artefact expressivity. Finally, we cluster work that emphasizes the interplay between human and artefact expressivity in interaction.

\subsection{Human Expressivity}

3.1.1 As Sensory-Motor Activity. Wensveen et al.'s alarm clock provides a means for expressing emotions by manipulating the sliders of the product, which then interprets these emotions from the movements and created patterns [76]. The underlying vision is that "Future interactive systems need to recognize emotional aspects in order to be truly adaptive" [77]. Expressivity in this interpretation typically involves gestures and actions towards a system, which then interprets the emotional state of the user.

3.1.2 As Dynamic Form. Human expressivity in HCI also focuses on how technology allows people to express bodily movement such as gestures and touch with the agenda of allowing for freedom of expression. This can be functionally, to improve interaction possibilities through higher input resolution, such as Marquardt et al.'s fiduciary tagged gloves: "The hand has incredible potential as an expressive input device" [45]. Along these lines Caramiaux et al. present an approach to detect gesture expressivity through muscle sensing enabling the tracking of subtle variations in how gestures are expressed. In their definition of expressivity, it "involves the idea of potential variation instantiated by the consistent constitutive structure", in this case of gestures [10]. Therefore, enabling technologies have been developed that can recognize a great variety of gestures [20]. In this line of thinking, the agenda is concerned generally with broadening the opportunities for input. This is also expressed by Benyon et al. who state that "expressive interaction focuses on the impact of speech, touch and gesture on opportunities for new forms of interaction" [2]. This relates to what Wensveen et al. named "Freedom of Interaction" [77].

3.1.3 As Social Activity. A related approach is to design systems allowing for human emotional expressivity, for human interpretation and not for system interpretation. This can be in the form of self-reflection, as in the case of Affective Diary, which analyses various types of behavioral and environmental data to support personal reflection [42]. Or in the form of supporting human-to-human communication as is the case with eMoto [20], InTouch [6] and Feather, Scent and Shaker [66]. eMoto allows people to communicate their emotions through a mobile phone application that adapts the imagery of a message by interpreting the gestures of its user
[20]. InTouch allows people to express their emotions by rolling a set of pins, the movement is then replicated when another manipulates a similar set of pins elsewhere [6]. In Strong and Gaver's terms "the concern is not to exchange information, but rather to express mood and emotion" [66]. They emphasize "the potential for technology to mediate interactions that are indicative rather than explicit, expressive rather than informative, and emotive rather than instrumental". These cases emphasize the value of ambiguity and subtleness.

3.1.4 As Value. A recurring perspective on human expressivity is that of allowing people to express themselves as part of a creative activity offering a valuable contribution, e.g. to an audience. The creation of music is a dominant case in this area often focusing on musical creation using tangible interfaces [35, 50, 69, 73]. Music is a fundamental human practice and research has explored the opportunities for e.g. supporting composers in developing their personal musical expressions by combining the expressive power of computation and paper [69], empowering children in making music collectively [35], having a shared interface (tabletop) for expressing musical ideas [51] or having a shape-changing interface inviting people to explore abstract and imperfect sounds [73]. Since 2001, there has been an annual conference on New Interfaces for Musical Expression (nime.org). In creative activities that aim for a goal, such as a musical composition, skill development starts to play an important role when interacting.

3.1.5 Summarizing Human Expressivity. Overall, human expressivity in HCI focuses on broadening the input space by involving a diversity of input modalities. The illustrated designs use various types of input, such as body movement, physiological data or contextual data, to analyze emotional content. The projects emphasize concepts such as freedom of interaction, i.e. allowing for diverse possibilities to express oneself [75] and ambiguity of information presentation [42], considering freedom of expression to communicate with the system. Researchers are therefore inspired by movement analysis techniques, such as Laban movement-analysis. Furthermore, there is an emphasis on how designers should become aware of and use their bodies to design for expressive, rich behavior by means of methods such as choreography of interaction [34]. This relates to the concept of somaesthetics which addresses the importance of bodily movements, sensations, and somatic training, as part of our ways of being and thinking in the aesthetic experience [30]. Given the exemplars, one could argue that to support expressive interaction, interfaces should be able to interpret a wide variety of user actions as system input, and users can learn to control the system's output through skill development. However, as this large variety of data is also difficult to analyze and interpret, the output mechanisms of the interface should also give room to allow for interpretation, which brings us to our second perspective, the artefact expressivity.

\subsection{Artefact Expressivity}

3.2.1 As Sensory-Motor Activity. Vallgårda and Sokoler discuss how different material properties such as tensile strength, opaqueness or thermal behavior can be manipulated by a system to allow for unique computational expressions. They propose a perspective 
of computational composites and emphasize that designers must familiarize themselves with the expressiveness of material form also when designing with computational technology [72]. Focus on this perspective is primarily on system expressivity towards humans by means of temporal form.

3.2.2 As Dynamic Form. Parkes et al. emphasize that physical kinetic motion of objects can be used to embody and communicate information [48]. This is illustrated by Lumen, an interactive display that presents physical and visual imagery through smooth and continuous movement, thereby providing aesthetically pleasing experience as it is being touched [53]. With Thrifty Faucet, Togler et al. explore how a water tap with life-like behavior can provide meaningful and vital expressions [68]. Both examples are responsive to human behavior in the form of providing multimodal forms of feedback.

3.2.3 As Social Activity. Deckers et al. developed a light object with perceptive and expressive qualities that express meaning to the user in the interaction [14]. This object goes a step further by actively responding to exploratory and expressive behaviors of the user in an expressive dialogue. This is in line with Topobo, a constructive toy with kinetic memory [55]. Raffle et al. describe one of the design principles underlying Topobo as "Be expressive encourage exploration of a topic without prescribing "right" and "wrong" activities" [55]. Both systems support an action-perception loop between user and system.

3.2.4 As Value. Finally, some see expressivity as a broader sense of the place things have in people's lives. For example, Hallnäs and Redström argue that "we have to design these computational everyday things in ways that makes it possible for people to give them meaning, to give them a place in their lives, in various ways" [27]. "However, a given thing can be used not only for doing certain things, it can be used also to express various things, such as our lifestyle, the values we believe in, the (sub)cultures we belong to, etc." [27]. Another example of this is Lynggaard et al. who studied how wealthy people stage themselves and their power through smart homes equipped with e.g. technology, which can appear and disappear on demand [43]. This places the system expressivity in the social or societal context of its actors.

3.2.5 Summarizing Artefact Expressivity. While for human expressivity, emphasis is on expressive gestures by the user, in contrast artefact expressivity is more related to the display qualities or output of the system. The exemplars show designs with different types of output, where many emphasize the motion component. One can argue that for a system or object to be expressive, dynamic qualities are key, and this is illustrated in considerations on temporal form [71] or as stated by Hallnäs and Redström: "the expressionidentity of computational artefacts is based on a combination of time structures (computation) and space structures (manipulation and display of results)" [27]. Richness of the movement qualities is also an important component of expressivity of the objects, which can be achieved both by diversity in modalities, but also through more complex qualities such as shape change [53]. Authors also emphasize aspects such as subtleness [14] and the development of new aesthetic qualities [71]. Deckers et al. consider how the reciprocal qualities of technology support the interplay between person and artifact [14], which connects to the third perspective, the expressivity in interaction. And finally, the social or societal context in which the artefact is used plays a role in the meaning that is attributed to its expressivity.

\section{EXPRESSIVITY IN INTERACTION: LITERATURE REVIEW AND FRAMEWORK}

Expressivity in interaction focuses on the interplay between both the human's and artifact's expressive actions to achieve a certain goal. While the above examples all deal with interactive technology, they focus primarily on either the human or the artefact side of the interaction. But we have also found a cluster of research artefacts which place emphasis on both sides and in particular, the interplay between them. This is for example reflected in the work of Ross and Wensveen who present a lamp that involves interaction by means of social sensitive bodily movements [61], Ross named this the unity of interaction [59]. Through the dynamic interplay of the user's gestures and the light response, both user and system find the socially most appropriate setting. Another example is the affective pen developed by Bruns Alonso et al. which seeks to reduce the nervous behaviors of its user. When the user expresses fidgeting behaviors towards the pen, its haptic properties change, for example by increasing the friction for a rotating movement. In this interplay of action and response, the user's nervous behaviors are reduced while the pen's properties are restored [7]. Finally, Jakobsen et al. designed a system aimed to empower children to collectively make expressive music. The children were provided with a LEGO-Mindstorms-based platform for constructing collective music instruments and using these instruments to control a complex musical back-end. This allowed children with very different levels of skills to engage with the instruments and to create their own music. In this work, emphasis was on empowering children to generate expressive instruments as well as musical expressions [35]. Thus, this implies a focus not only on the interaction, but also on the outcome of the interaction. Dobrian and Koppelman argue that, sophisticated musical expressions not just require control but virtuosic mastery of the instrument or interface and that the instrument is not expressive, but can be used to transmit human expressiveness when it allows for skill development [17].

Aesthetics of interaction is frequently used to describe the expressive interplay between the user and the artefact $[35,61]$. Herewith, the reciprocal qualities described by Deckers et al. [14] are taken a step further; creating an action-perception loop that can be initiated by the expressive behavior of both the user and the artifact. While the perspectives of expressivity from a human, artefact and interaction perspective are not mutually exclusive nor opposites, they do represent different emphasis. In the expressivity of the interaction, the elements that were described in both the expressivity of the human and the expressivity of the artefact are combined.

\subsection{Learning from Research Artefacts through a Literature Review of Design Rationales}

Over the past decades, the field of HCI has increasingly included design as a research discipline that contributes knowledge through the construction of artefacts [23]. Learning from research artefacts 

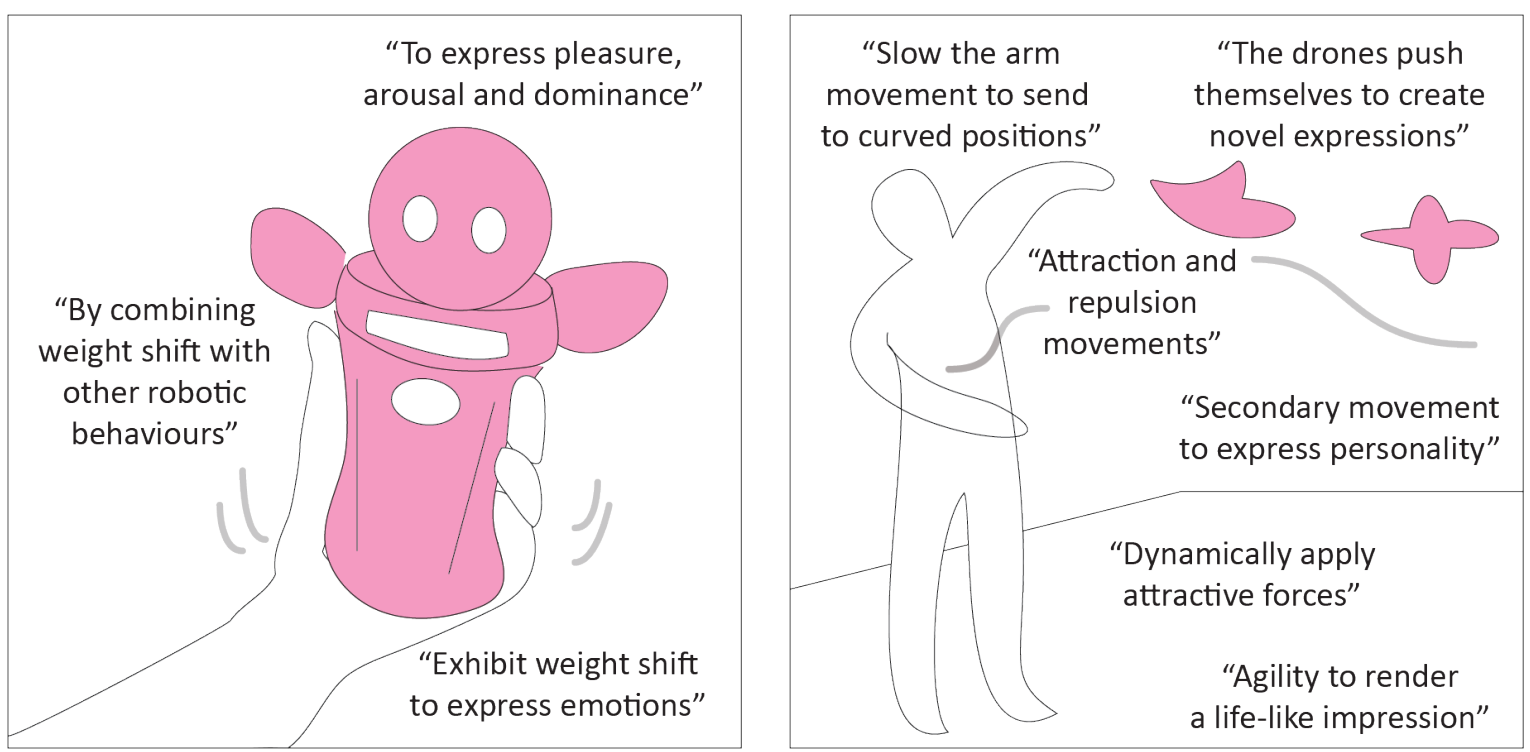

Figure 4: Annotated sketches of the design cases OMOY [46] and Dancing with Drones [19], including the author's statements related to expressivity in interaction.

in this context has two purposes. First, as expressivity is an emerging term which has not yet been conceptually and theoretically agreed upon in the field of HCI, we can learn from the knowledge which is embedded in individual artefacts, while not yet matured across the field. Second, by learning from the design of artefacts we can provide inspiration for designers in designing for expressivity in interaction by combining and reflecting upon a collection of exemplars. In design there is a long tradition in learning about phenomena and inspiring new designs through exemplars [e.g. $12,16,25]$. In addition, it has been pointed out how HCI-frameworks often fail to relate to specific trade-offs that designers are working with and therefore become less operational when informing design [2].

In the field of $\mathrm{HCI}$ we are beginning to see various forms of metaanalyses [e.g. 1,12,24,31]. On the one hand we find meta-analyses that are inspired by research in medicine and related research areas, primarily quantitative analyses of huge collections of research papers. On the other hand, and closer to research artefacts that come out of design research or research-through-design, we see formats such as meta-analyses across a small collection of research artefacts of one author or research group [12] or the format of annotated portfolio which comprises a small collection of research artefacts of different origins [24]. Illustrating how theory can be developed based on research-through-design work, Gaver argues:

"Conceptual work appears in many forms as a routine part of research-through-design work. Whenever practitioners describe their influences, discuss the rationales for design decisions, and articulate their assessment of what they have made and its importance, they engage in a form of implicit conceptual work by highlighting important issues, dimensions of similarity, and criteria for choices and success. To the extent these conceptual statements are articulated in general terms and applied to multiple examples, they become recognizable as theories in their own right" [23, p938].

Artefacts as theory nexus [11] is another way of phrasing a similar argument. In this paper we combine these methodologies by identifying papers that engage with expressivity in some sense related to interaction. From this selection of papers, we identify research prototypes and elicit their rationales from the writing, through annotations on the developed portfolio. By clustering these annotations, we identify common concepts articulating expressivity in interaction. In this way contributing to $\mathrm{HCI}$ theory in terms of the framework presented in figure 5

4.1.1 Literature review. Having identified the different perspectives on expressivity in HCI, we conducted a systematic and broader review to investigate how these perspectives are represented more broadly in the HCI field. We scoped the analysis on publications in the three key ACM conferences relating to design (CHI, TEI and DIS) to investigate current conceptions of expressivity from a representative rather than exhaustive sample. Within the ACM Digital Library, we identified 251 publications that matched all the terms: "express"” AND "interact*" AND "design" in their abstract during the period from 2016 to 2020 .

Of these papers, 36 demo papers, which were also presented as a paper, courses, panels, SIGs, workshops, video showcases and doctoral consortia, were excluded from our selection. We reviewed all remaining abstracts and removed papers in which "express*" was used in the linguistic sense as a synonym to words such as "articulate" or "signify" or the term "expression" was used to indicate a "word" or "phrase". We also removed papers in which derivatives form "express" were not used to address interaction with a system presented by the authors. In most cases "expression" or "express" 
was used to make generic statements relating to emotions and bodily movements, or it addressed concepts such as self-expression, creative expression, expressive potential or expressive power. This analysis resulted in a selection of 39 papers that presented a design case. After analyzing the descriptions of the design cases, we excluded 10 papers that only described the design of input (9) or output (1), resulting in a final selection of 29 publications presenting a design case addressing expressivity in interaction.

4.1.2 Analysis of Design Cases. In this final analysis, we investigate the design artefacts and rationales from the sample papers more closely, to understand how to design for expressivity in interaction and to further develop a framework for expressivity in interaction. We reviewed our selection of papers and extracted the explanations of how expressivity in interaction was designed. From these descriptions, we annotated the authors' statements in which an aspect of the interaction was linked to expressivity in all its respective definitions, often a publication included multiple statements (see e.g. figure 4). We collected a total of 85 statements linking to expressivity in interaction and used thematic analysis to cluster them into categories, building on the definitions we had found in our review of perspectives (emotional state, freedom of interaction, ambiguity, subtleness, skill development, temporal form, multimodality, action-perception loops and societal context). This process was done separately by two authors and inconsistencies were discussed and agreed upon by the authors in a combined clustering event.

4.1.3 Results. Most papers include statements that link expressivity with (human) emotional qualities, often by adding an interface to reproduce human expression. A robot equipped with an expressive face [81], a facial expression for an animation or experiences that coincide with anthropomorphic interactivity [40]. Another way to express emotional qualities was through movement. For example, using weight shift to express emotions [46], or material change as novel robotic behavior [33] or emotive behavior through motion [15]. In some cases, the objects are self-learning from their context [21] or the interaction with the user [15, 81, 84]. However, not all projects use anthropomorphic qualities to evoke emotional qualities, some generate a change of properties such as "expressive lights", "blend texture and color with context" or "symbolizing human relationship" to indicate emotions [28, 39, 40, 58, 63].

The second group of statements addressed temporal form. For example, "the entire surface as tangible interface", "playing with the temporal from" or "the drones push themselves to create novel expressions" [3, 4, 18, 19, 82, 83]. Some statements relate to moldable qualities or limitations e.g. "deformable interfaces for expressive therapy" or "limiting movement to enlarge impact" [5, 38, 45, 82], others stress the organic nature of shape change through "agility to render life-like impression" or "giving a sense of organic matter" [4, 19]. The temporal form was often considered as multi-interpretable or ambiguous “encouraging interpretation' or "evoking something unfamiliar" [3, 38-40, 57, 63, 82].

Statements linking to expressivity in interaction, which can be found amongst most projects, are those related to multimodal qualities. These statements emphasize that a plurality of interactions is more likely to create an expressive environment $[33,37,40,45,46,62,63,79,82]$. Some projects extend the notion of multimodal qualities to enhance human expressivity [41, 74] or the possibility to interact with intangible data [83]. We also found statements linked to the action-perception loops in which the interactivity is central to the expressivity. Most projects focus on the interaction related to the object, e.g. "a dance performance with aesthetic harmony", "being alive engagement in the world" or "attraction and repulsion movements" [5, 19, 28, 38, 39, 80, 83], some emphasize the inter-human relationship [33, 79] in which the object supports collaboration.

Similar collaboration can be found amongst the statements which highlight the expressivity by building a relationship through skill development, in which experience over time plays a significant role. These skills can relate to the object, comparable with musical instruments or tools $[15,37,57,62,74]$ or to a collaboration with multiple users $[28,33,74]$. When focusing on the action itself, some statements explained the expressivity as relating to subtlety explaining it through the nuance of certain actions $[5,8,19]$. Finally, statements regarded the freedom of interaction or the ability to interact in multiple ways [33, 41, 45, 46, 62, 79, 84].

\subsection{A Framework for Expressivity in Interaction}

With the confirmation of the qualities that determine expressivity in interaction, through the literature survey, we propose a Framework for Expressivity in Interaction (see figure 5). The framework considers the ability of humans to express their emotional state towards a system through a variety of (inter)actions and behaviors. They do this in an action-perception loop with the system or artefact as embodiment of the system, which changes its form and materiality over time, in response to the user's actions. These changes can relate to different modalities, both from the human (body movement, speech etc.) as well as from the artifact (sounds, colors, shape-change, taste etc.). The changes can have different levels of nuance, as the system should be able to perceive subtle actions of the user but should also be able to change its form in subtle ways. Expressive interaction should leave room for ambiguity, as the mapping between action and perception cannot always be directly coupled, which will support engagement and skill development. Humans can learn to control the system's output, but the system can also potentially learn about the human and guide their actions or understand their preferences. When designing for expressive interaction, designers can take these qualities into account to generate expressive interfaces or evaluate the expressivity of their design. In addition, they can consider the socio-cultural setting in which the interaction will take place. The setting can involve multiple humans, connected artefacts and consideration may need to be given at how humans and artifacts in such setting communicate and/or relate to each other, e.g. regarding the outcomes of an interaction when playing music.

In summary, expressivity in interaction refers to the quality of being expressive, i.e. the ability to effectively convey thought or feeling by displaying an emotional state through verbal and/or nonverbal behavior. Human expressive actions constitute freedom of interaction and emotional expressivity; artifact expressive actions are achieved by its temporal form. The interplay addresses multimodal qualities, subtlety and ambiguity. Through the interplay a skill can be developed towards achieving a certain goal. For the 


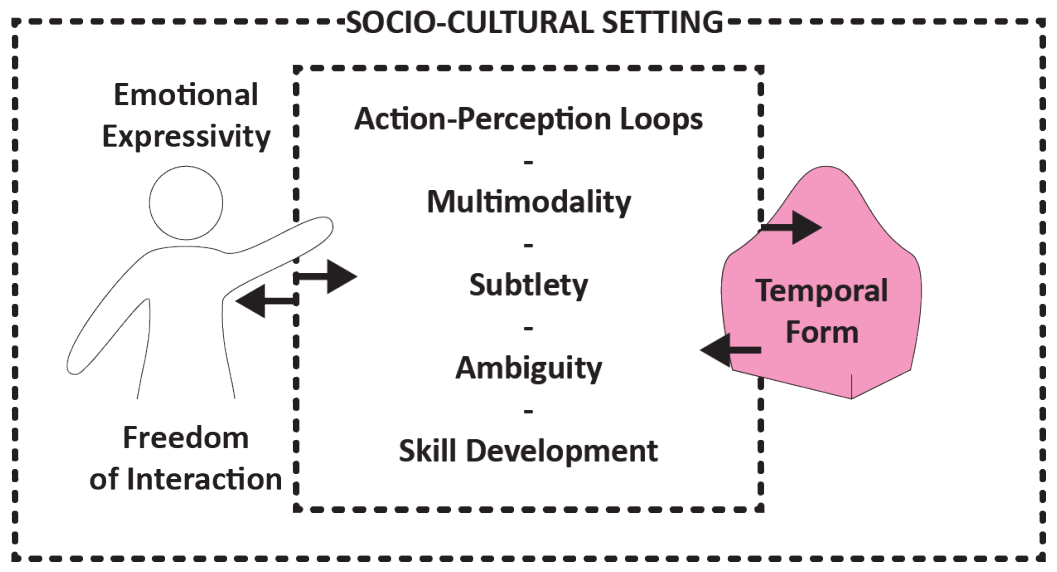

Figure 5: Graphic representation of the Framework for Expressivity in Interaction, highlighting the different qualities that designers can employ when designing for expressive interaction.

nature of expressivity in interaction we elaborate on the context of musical compositions, in which expressive interaction is a mechanism, where expression is achieved through subtle and rich cues by a performer and its decoding by a receiver in real-time, giving the performance a trajectory [17]. In the context of this paper, human and artefact can be both performer and receiver. Although artefacts in themselves do not have feelings, they can be used to transmit expressivity by capturing data, and providing finely detailed and complex correspondences between input and output, the action-perception-loops between human and artefact.

\section{FRANK AN EXPRESSIVE SOFT-ROBOTIC SHAPE-CHANGING INTERFACE}

Having derived the framework for expressivity in interaction based on analyzing existing definitions, artefacts and design rationales, we deployed the framework in a masters course on interactive materiality to provide an example of how the framework can inform design. This worked placed emphasis on how designers can explore and emphasize the proposed set of qualities of the framework to design for expressivity in interaction. In this section we present one of the resulting design cases of a shape-changing soft-robotic interface FRANK, to illustrate how our framework can also be used as a generative tool (in addition to analyzing existing designs).

FRANK was designed by a group of four master level interaction design students Davide Jose Nogueira Amorim, Giulia Caleca, Minerva Loos and Alex de Ruiter and presented at ACM TEI'19 student design challenge. FRANK was developed, by going through material centered design process during "Interactive Materiality", an eightweek Master course (5 ECTS, $\pm 14 \mathrm{~h} / \mathrm{w}$ ) offered at the Department of Industrial Design of Eindhoven University of Technology by Miguel Bruns and Simone de Waart. The course focuses on learning about design theory by applying acquired knowledge regarding novel materials and aesthetics of interaction in a research-throughdesign artefact. The students first received an introduction into (smart) material developments, the material turn in HCI, as well as into shape-changing interfaces and aesthetics of interaction. Consequently, they explored expressive human movements and matching material qualities in a hands-on workshop led by material designer Simone de Waart. Finally, the framework for Expressivity in Interaction was presented by the first author and they had to design an interactive material experience, following a process of analyzing (affirming and appreciating the human movements), synthesizing (mapping human movements to an artefact behavior), and detailing (fine-tuning the dynamic form of the artefact) [65].

The prototype named FRANK (figure 6) is an interactive surface composed of rubbery hairy elements, which together create a novel material experience. Like Cilllia [47], a project that inspired the student group, the combined effect of the single hairs defines the user experience creating a novel type of tactile experience with an inherent aesthetic quality. It was inspired by the aesthetic and behavioral properties of natural materials, such as the multi-element structure of grass and the softness and pliability of mushrooms. FRANK allows for exploring the behavioral opportunities of a programmable interactive material. The system is composed of a $7 x 7$ grid of embedded capacitive copper wire touch sensors, a brushless DC Gimbal Motor (GM4008H) which was connected to three equally spaced parallel axes using a spur gear mechanism to create a wave movement in the surface. Two microcontrollers (Arduino UNO for input and DUE for output) were connected with a serial connection to program its behavior in detail.

"FRANK is an interactive material experience that senses and reacts to user's input. By embedding sensors, magnetic actuators and a microcontroller, we were able to program its behavior in detail. The interaction flow between the user and FRANK (shown in fig. 7) is as follows: when no users are close to interact, FRANK moves in what we will call 'normal' speed. Through this kind of augmented feedforward (as described by Wensveen et al., in [75]) the material invites people to interact with it and follow its movement with their hands. The nuanced movements of the single hairs, caused by the actuated movement, adds a layer of inherent feedforward, which gives users a hint about the material properties and how it reacts 


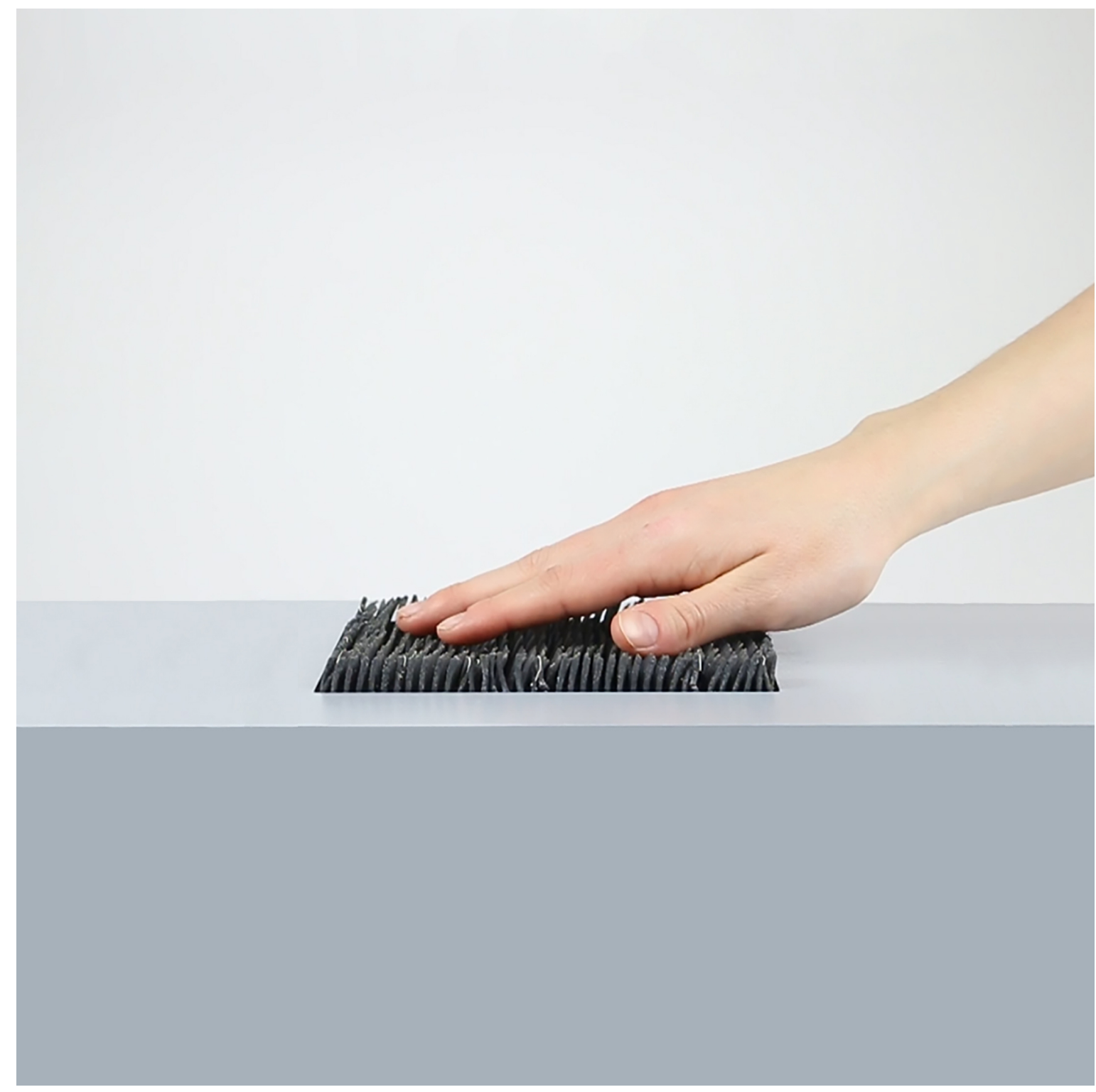

Figure 6: Hand touching FRANK (photo by Nogueira Amorim, Caleca, Loos and De Ruiter).

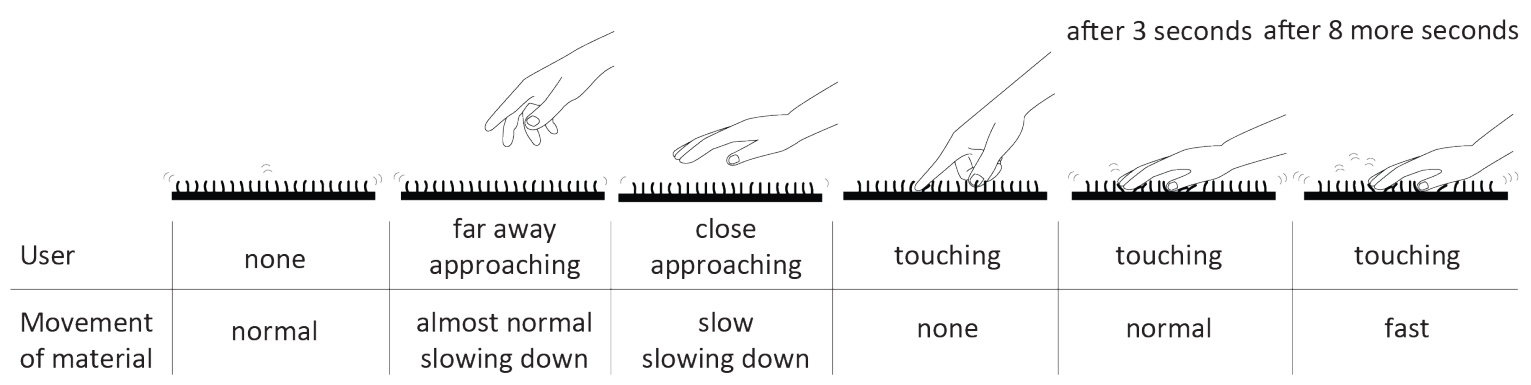

Figure 7: Behavior of FRANK in interaction with user, a video can be found under: https://vimeo.com/259247002 (illustration by Nogueira Amorim, Caleca, Loos and De Ruiter). 
to movements. As soon as the presence of a person is detected, the movement speed adapts to the distance of the person: the closer users get to the surface with their hands, the slower the movement becomes. As soon as users touch the surface, the movement stops. By doing so, the surface expresses a 'shy' invitation to be explored and to experience the inherent tactile qualities of the material without interfering with the users' explorative movements. This gives the users a moment to feel the malleability of each single 'hair' by going through them with their fingers. This moment lasts 3 seconds, after which the movement of the motor starts again, adding an augmented feedback level to the inherent material feedback. The system allows for this interaction for another 8 seconds. Then, suddenly, the speed of movement increases. The high speed rotation continues until the user take its hand off the surface. At this point, the material starts to move at normal speed, inviting again to be touched." [Nogueira Amorim, Caleca, Loos and De Ruiter describing the interaction in their TEI 2019 Student Design Challenge - submission "FRANK: An Exploration into the Potential of Expressive and Engaging Robotic Materials"]

FRANK affords subtle and consequently emotionally expressive hand-movements. Users can for example express calmness through caressing movements or annoyance by playing with a single hair, thereby allowing for freedom of interaction. The designers carefully considered the connection between the users' action-perception loop and the temporal form of the material surface (as illustrated in figure 7). In addition to the subtle movements by the user, the material surface also provides different nuanced and sometimes ambiguous responses to the user's actions, e.g. when the material is "tired" of it being touched. The interaction with the surface is short, and the exercise was not intended at considering a specific context as the course focused on design theory rather than skill application.

\section{DISCUSSION}

This paper took a close look at the concept of expressivity in interaction and proposes a Framework for Expressivity in Interaction as its main contribution. The framework was developed employing a multi-method approach. It first addressed the different definitions of expressivity followed by perspectives that are taken when considering expressivity, studied exemplar research-through-design cases to define what qualities make up expressivity in interaction, and validated the qualities by means of a literature review investigating all design cases published in the three main design-related conferences in the field of $\mathrm{HCI}$ over the past five years. A design case of a shape-changing soft-robotic interface was developed using the qualities of the framework to illustrate how designers can make use of it in their practice. Given the extensive discussion regarding expressivity in interaction throughout the paper, we will focus our discussion on the limitations of our work and provide some insights for further development.
Our work was triggered by the emergence of projects building of novel forms of interaction involving shape-change [54] and materiality [26] that claim expressivity and it was inspired by a variety of research-through-design projects that address expressivity. The separation into human-, artefact- and interaction expressivity seems artificial and does not acknowledge the holistic perspective that is generally considered in these design cases. Also, it sometimes was difficult to clearly distinguish the category in which some projects fall. However, making the distinction helped in structuring the argument and identifying the qualities that define expressivity. In the end, the values are presented as being part of the interaction in the framework, addressing both human and artefact, with the exception of the qualities that address the human (emotional expressivity and freedom of interaction) or the artefact (temporal form).

In the preparation of our research, we provided an extensive perspective on expressivity. Our analysis of research-through-design cases included research projects acquired through the full ACM library on computing literature, design journals and IEEE conferences. For our review, we decided to scope our selection to a manageable format focusing only on designs presented at the ACM conferences CHI, TEI and DIS. Other conferences in the ACM Digital Library yielded limited to no results for which we decided to not include them in our analysis. In addition, we believe that the three conferences considered are well-known for accepting design cases as contributions. The exclusion of papers was based on their abstracts and using "express" ${ }^{*}$ as a search term may have limited the outcomes of our review. Although other words may be related to the concept of expressivity, we considered that this term best covered our intentions based on the analysis of the works by Forlizzi and Battarbee [22], Wensveen et al. [75] and Dalsgaard et al. [12]. In the end, the intention of the review was not to be exhaustive but meant to validate the qualities which we had observed in the analysis of research-through-design cases.

We illustrated how our framework was used to inform the design of a soft-robotic interface in a master level design course. A clear limitation was that skill development was not considered in the current design, which is a general concern with research artefacts addressing expressivity in interaction which are mostly presented as a proof-of-concept demonstrations of a new design [17]. On the other hand, follow-up discussions reflected the possibility of incorporating this type of interaction in contexts such as human-vehicle interaction, e.g. to prevent visual distraction while supporting the selection of music. Future work in such settings or in contexts such as musical expression can support further research in relation to skill development. In addition, while the presented guidelines can support designers, further research is required into the extent to which all (or a selection of) qualities should be incorporated to achieve expressivity in interaction.

The framework presented supports in clarifying some of the key concepts involved in designing for expressivity in interaction. But still, there are many things to be explored in relation to expressivity in interaction such as the connection to the aesthetics of interaction $[51,59]$ and the focus on skill development in contexts such as music composition [17]. Also, one may consider that the framework oversimplifies highly complex processes when moving beyond the one-person-one-artifact perspective, as is already shown in the project Dancing with Drones [19]. Developments in technology 
regarding robot swarms or the Internet-of-Things, where artefacts start to become part of an ecology [36], will require the extension of our framework. In such settings multiple people interact with multiple artefacts, or artefacts will start to interact with each other. We hint at this by presenting the socio-cultural section in which the interaction takes place, but this will require further investigation.

We want to end our discussion with a reflection on the designerly approach we took towards composing this paper and the analysis of our literature review. By selecting research-through-design artefacts, reviewing design cases and creating annotations on the design cases presented by others, we realized how complex it is to understand the rationale of some designers. This oftentimes implied the need for some sort of interpretation of the data presented in the papers. However, we believe that this exercise has also shown a novel approach to a meta-analysis which can contribute knowledge to the design discipline. Through our focus on how artefacts were designed, we show a novel approach of using artefacts as theory nexus [11].

\section{CONCLUSION}

We have offered a broad perspective on the use of expressivity in the context of $\mathrm{HCI}$, which we believe will become increasingly important due to improved sensing technologies and more complex means of actuation. Through a literature review and the analysis of various design cases, we contribute to the knowledge development relating to expressivity in $\mathrm{HCI}$ and developed a Framework for Expressivity in Interaction. The framework intends to highlight salient aspects when designing for expressivity in interaction. It combines insights from well-known concepts in design such as the freedom of interaction [75], action-perception loops, multimodality, subtlety, ambiguity [25], skill development and temporal form [71]. Our work brought together new perspectives on expressivity in interaction for which we argue that expressivity in interaction should be considered a type of interaction as discussed by Hornbæk and Oulusvirta [32]. In a time where artefacts become increasingly dynamic, our framework can support designers in considering which qualities can make interaction more expressive.

\section{ACKNOWLEDGMENTS}

We would to thank Davide Jose Nogueira Amorim, Giulia Caleca, Minerva Loos and Alex de Ruiter for allowing us to use their design project as a case for our paper and Simone de Waart for enabling us to test our framework in the "Interactive Materiality" course and contributing to the design case through her coaching and expertise.

\section{REFERENCES}

[1] Ashraf Abdul, Jo Vermeulen, Danding Wang, Brian Y. Lim, and Mohan Kankanhalli. 2018. Trends and Trajectories for Explainable, Accountable and Intelligible Systems: An HCI Research Agenda. In Proceedings of the $2018 \mathrm{CHI}$ Conference on Human Factors in Computing Systems (CHI '18). Association for Computing Machinery, New York, NY, USA, Paper 582, 18 pages. DOI: https://doi.org/10.1145/3173574.3174156

[2] David Benyon, Kristina Höök, and Laurence Nigay. 2010. Spaces of Interaction In Proceedings of the 2010 ACM-BCS Visions of Computer Science Conference (ACM-BCS '10). Association for Computing Machinery, New York, NY, USA, 2:1-2:7. Retrieved from http://dl.acm.org/citation.cfm?id=1811182.1811185

[3] Harvey Bewley and Laurens Boer. 2018. Designing Blo-nut: Design Principles, Choreography and Otherness in an Expressive Social Robot. In Proceedings of the 2018 Designing Interactive Systems Conference (DIS '18). Association for
Computing Machinery, New York, NY, USA, 1069-1080. DOI: https://doi.org/10. 1145/3196709.3196817

[4] Harvey Bewley and Anna Vallgårda. 2017. Provoking Performing Objects: Expanding the Design Space for Soft Robotics. In Proceedings of the 2017 ACM Conference Companion Publication on Designing Interactive Systems (DIS '17 Companion). Association for Computing Machinery, New York, NY, USA, 243248. https://doi.org/10.1145/3064857.3079154

[5] Alberto Boem and Giovanni Maria Troiano. 2019. Non-Rigid HCI: A Review of Deformable Interfaces and Input. In Proceedings of the 2019 on Designing Interactive Systems Conference (DIS '19). Association for Computing Machinery, New York, NY, USA, 885-906. DOI: https://doi.org/10.1145/3322276.3322347

[6] Scott Brave and Andrew Dahley. 1997. inTouch: A Medium for Haptic Interpersonal Communication. In CHI ' 97 Extended Abstracts on Human Factors in Computing Systems (CHI EA '97). Association for Computing Machinery, New York, NY, USA, 363-364. https://doi.org/10.1145/1120212.1120435

[7] Miguel Bruns Alonso, Caroline C. Hummels, David V. Keyson, and Paul P. Hekkert. 2013. Measuring and Adapting Behavior During Product Interaction to Influence Affect. Personal Ubiquitous Computing 17, 1: 81-91. https://doi.org/10.1007/ s00779-011-0472-3

[8] Wei Cai, Anand Doshi, Chelsea Lauren Miller, and Shu Zhou. 2018. Musi: Facilitating Asynchronous Practice Between Classical Musicians. In Extended Abstracts of the 2018 CHI Conference on Human Factors in Computing Systems (CHI EA '18). Association for Computing Machinery, New York, NY, USA, Paper SDC06, 1-6. DOI: https://doi.org/10.1145/3170427.3180648

[9] Antonio Camurri, Gualtiero Volpe, Giovanni De Poli, and Marc Leman. 2005. Communicating Expressiveness and Affect in Multimodal Interactive Systems. IEEE MultiMedia 12, 1: 43-53. https://doi.org/10.1109/MMUL.2005.2

[10] Baptiste Caramiaux, Marco Donnarumma, and Atau Tanaka. 2015. Understanding Gesture Expressivity through Muscle Sensing. ACM Transactions on ComputerHuman Interaction 21, 6: 1-26. https://doi.org/10.1145/2687922

[11] J. M. Carroll and W. A. Kellogg. 1989. Artifact As Theory-nexus: Hermeneutics Meets Theory-based Design. In Proceedings of the SIGCHI Conference on Human Factors in Computing Systems (CHI '89). Association for Computing Machinery, New York, NY, USA, 7-14. https://doi.org/10.1145/67449.67452

[12] Peter Dalsgaard, Kim Halskov, and Ole Sejer Iversen. 2016. Participation Gestalt: Analysing Participatory Qualities of Interaction in Public Space. In Proceedings of the 2016 CHI Conference on Human Factors in Computing Systems (CHI '16). Association for Computing Machinery, New York, NY, USA, 4435-4446. https://doi.org/10.1145/2858036.2858147

[13] Peter Dalsgaard and Lone Koefoed Hansen. 2008. Performing perception-staging aesthetics of interaction. ACM Transactions on Computer-Human Interaction 15, 3: 1-33. https://doi.org/10.1145/1453152.1453156

[14] Eva Deckers, Stephan Wensveen, Rene Ahn, and Kees Overbeeke. 2011. Designing for Perceptual Crossing to Improve User Involvement. In Proceedings of the SIGCHI Conference on Human Factors in Computing Systems (CHI '11). Association for Computing Machinery, New York, NY, USA, 1929-1938. https://doi.org/10.1145/1978942.1979222

[15] Ruta Desai, Fraser Anderson, Justin Matejka, Stelian Coros, James McCann, George Fitzmaurice, and Tovi Grossman. 2019. Geppetto: Enabling Semantic Design of Expressive Robot Behaviors. In Proceedings of the 2019 CHI Conference on Human Factors in Computing Systems (CHI '19). Association for Computing Machinery, New York, NY, USA, Paper 369, 1-14. DOI: https://doi.org/10.1145/ 3290605.3300599

[16] Tom Djajadiningrat, Stephan Wensveen, Joep Frens, and Kees Overbeeke. 2004. Tangible Products: Redressing the Balance Between Appearance and Action. Personal Ubiquitous Computing 8, 5: 294-309. https://doi.org/10.1007/s00779004-0293-8

[17] Christopher Dobrian and Daniel Koppelman. 2006. The 'E' in NIME: musical expression with new computer interfaces. In Proceedings of the 2006 conference on New interfaces for musical expression (NIME '06). IRCAM - Centre Pompidou, Paris, FRA, 277-282.

[18] Soya Eguchi, Ryoma Takamori, Ryohei Yuasa, and Hiroya Tanaka. 2019. Morphing Design for Socially Interactive Autonomous Car by Multi-Material 3D-Printing. In Extended Abstracts of the 2019 CHI Conference on Human Factors in Computing Systems (CHI EA '19). Association for Computing Machinery, New York, NY, USA, Paper LBW1310, 1-6. DOI: https://doi.org/10.1145/3290607.3312939

[19] Sara Eriksson, Åsa Unander-Scharin, Vincent Trichon, Carl Unander-Scharin, Hedvig Kjellström, and Kristina Höök. 2019. Dancing With Drones: Crafting Novel Artistic Expressions Through Intercorporeality. In Proceedings of the 2019 CHI Conference on Human Factors in Computing Systems (CHI '19). Association for Computing Machinery, New York, NY, USA, Paper 617, 1-12. DOI: https: //doi.org/10.1145/3290605.3300847

[20] Petra Fagerberg, Anna Ståhl, and Kristina Höök. 2004. eMoto: Emotionally Engaging Interaction. Personal Ubiquitous Computing 8, 5: 377-381. https: //doi.org/10.1007/s00779-004-0301-z

[21] Min Fan, Jianyu Fan, Sheng Jin, Alissa N. Antle, and Philippe Pasquier. 2018. EmoStory: A Game-based System Supporting Children's Emotional Development. In Extended Abstracts of the 2018 CHI Conference on Human Factors in 
Computing Systems (CHI EA '18). Association for Computing Machinery, New York, NY, USA, LBW058:1-LBW058:6. https://doi.org/10.1145/3170427.3188594

[22] Jodi Forlizzi and Katja Battarbee. 2004. Understanding Experience in Interactive Systems. In Proceedings of the 5th Conference on Designing Interactive Systems Processes, Practices, Methods, and Techniques (DIS '04). Association for Computing Machinery, New York, NY, USA, 261-268. https://doi.org/10.1145/1013115 1013152

[23] William Gaver. 2012. What Should We Expect from Research Through Design? In Proceedings of the SIGCHI Conference on Human Factors in Computing Systems (CHI '12). Association for Computing Machinery, New York, NY, USA, 937-946. https://doi.org/10.1145/2207676.2208538

[24] Willian Gaver and John Bowers. 2012. Annotated Portfolios. Interactions 19, 4: 40-49. https://doi.org/10.1145/2212877.2212889

[25] William Gaver, Jacob Beaver, and Steve Benford. 2003. Ambiguity As a Resource for Design. In Proceedings of the SIGCHI Conference on Human Factors in Computing Systems (CHI '03). Association for Computing Machinery, New York, NY, USA, 233-240. https://doi.org/10.1145/642611.642653

[26] Elisa Giaccardi and Elvin Karana. 2015. Foundations of Materials Experience: An Approach for HCI. In Proceedings of the 33rd Annual ACM Conference on Human Factors in Computing Systems (CHI '15). Association for Computing Machinery, New York, NY, USA, 2447-2456. DOI: https://doi.org/10.1145/2702123.2702337

[27] Lars Hallnäs and Johan Redström. 2002. From Use to Presence: On the Expressions and Aesthetics of Everyday Computational Things. ACM Transactions on Computer-Human Interaction 9, 2: 106-124. https://doi.org/10.1145/513665. 513668

[28] Heesoon Kim and James A. Landay. 2018. Aeroquake: Drone Augmented Dance. In Proceedings of the 2018 Designing Interactive Systems Conference (DIS '18) Association for Computing Machinery, New York, NY, USA, 691-701. DOI: https: //doi.org/10.1145/3196709.3196798

[29] Jonathan Hook, David Green, John McCarthy, Stuart Taylor, Peter Wright, and Patrick Olivier. 2011. A VJ Centered Exploration of Expressive Interaction. In Proceedings of the SIGCHI Conference on Human Factors in Computing Systems (CHI '11). Association for Computing Machinery, New York, NY, USA, 1265-1274. https://doi.org/10.1145/1978942.1979130

[30] Kristina Höök, Anna Ståhl, Martin Jonsson, Johanna Mercurio, Anna Karlsson, and Eva-Carin Banka Johnson. 2015. COVER STORY: Somaesthetic Design. Interactions 22, 4: 26-33. https://doi.org/10.1145/2770888

[31] Kasper Hornbæk and Morten Hertzum. 2017. Technology Acceptance and User Experience: A Review of the Experiential Component in HCI. ACM Transactions on Computer-Human Interaction 24, 5, Article 33 (October 2017), 30 pages. DOI https://doi.org/10.1145/3127358

[32] Kasper Hornbæk and Antti Oulasvirta. 2017. What Is Interaction? In Proceedings of the 2017 CHI Conference on Human Factors in Computing Systems (CHI '17). Association for Computing Machinery, New York, NY, USA, 5040-5052. https://doi.org/10.1145/3025453.3025765

[33] Maarten Houben, Benjamin Lehn, Noa van den Brink, Sabeth Diks, Jasmijn Verhoef, and Rens Brankaert. 2020. Turnaround: Exploring Care Relations in Dementia Through Design. In Extended Abstracts of the 2020 CHI Conference on Human Factors in Computing Systems (CHI EA '20). Association for Computing Machinery, New York, NY, USA, 1-8. DOI: https://doi.org/10.1145/3334480.3382846

[34] Caroline Hummels, Kees Overbeeke, and Sietske Klooster. 2007. Move to Get Moved: A Search for Methods, Tools and Knowledge to Design for Expressive and Rich Movement-based Interaction. Personal Ubiquitous Computing. 11, 8 677-690. https://doi.org/10.1007/s00779-006-0135-y

[35] Kasper Buhl Jakobsen, Jeppe Stougaard, Marianne Graves Petersen, Jakob Winge, Jens Emil and Majken Kirkegaard Rasmussen. 2016. Expressivity in Open-ended Constructive Play: Building and Playing Musical Lego Instruments. In Proceedings of the The 15th International Conference on Interaction Design and Children (IDC '16). Association for Computing Machinery, New York, NY, USA, 46-57. https://doi.org/10.1145/2930674.2930683

[36] Heekyoung Jung, Erik Stolterman, Will Ryan, Tonya Thompson, and Marty Siegel. 2008. Toward a framework for ecologies of artifacts: how are digital artifacts interconnected within a personal life? In Proceedings of the 5th Nordic conference on Human-computer interaction: building bridges (NordiCHI '08). Association for Computing Machinery, New York, NY, USA, 201-210. DOI: https: //doi.org/10.1145/1463160.1463182

[37] Takanori Komatsu, Kazuki Kobayashi, Seiji Yamada, Kotaro Funakoshi, and Mikio Nakano. 2018. Vibrational Artificial Subtle Expressions: Conveying System's Confidence Level to Users by Means of Smartphone Vibration. Proceedings of the $2018 \mathrm{CHI}$ Conference on Human Factors in Computing Systems. Association for Computing Machinery, New York, NY, USA, Paper 478, 1-9. DOI: https //doi.org/10.1145/3173574.3174052

[38] Pavel Karpashevich, Eva Hornecker, Michaela Honauer, and Pedro Sanches. 2018 Reinterpreting Schlemmer's Triadic Ballet: Interactive Costume for Unthinkable Movements. In Proceedings of the 2018 CHI Conference on Human Factors in Computing Systems (CHI '18). Association for Computing Machinery, New York, NY, USA, 61:1-61:13. https://doi.org/10.1145/3173574.3173635

[39] Young Suk Lee. 2017. Tea with Crows: Experiencing Proactive Ubiquitous Technology by Interactive Art. In Proceedings of the Eleventh International Conference on Tangible, Embedded, and Embodied Interaction (TEI '17). Association for Computing Machinery, New York, NY, USA, 677-680. https://doi.org/10.1145/ 3024969.3025058

[40] Young Suk Lee. 2018. Thou and I: Exploring Expressive Digital Interaction with Interactive Characteristic Wigs. In Proceedings of the Twelfth International Conference on Tangible, Embedded, and Embodied Interaction (TEI '18). Association for Computing Machinery, New York, NY, USA), 581-585. https://doi.org/10.1145/3173225.3173311

[41] Joanne Leong, Yuehan Wang, Romy Sayah, Stella Rossikopoulou Pappa, Florian Perteneder, and Hiroshi Ishii. 2019. SociaBowl: A Dynamic Table Centerpiece to Mediate Group Conversations. In Extended Abstracts of the $2019 \mathrm{CHI}$ Conference on Human Factors in Computing Systems (CHI EA '19). Association for Computing Machinery, New York, NY, USA, Paper LBW1114, 1-6. DOI https://doi.org/10.1145/3290607.3312775

[42] Madelene Lindström, Anna Ståhl, Kristina Höök, Petra Sundström, Jarmo Laaksolathi, Marco Combetto, Alex Taylor, and Roberto Bresin. 2006. Affective Diary: Designing for Bodily Expressiveness and Self-reflection. In CHI '06 Extended Abstracts on Human Factors in Computing Systems (CHI EA '06). Association for Computing Machinery, New York, NY, USA, 1037-1042. https: //doi.org/10.1145/1125451.1125649

[43] Aviaja Borup Lynggaard, Marianne Graves Petersen, and Sam Hepworth. 2012. "I Had a Dream and I Built It": Power and Self-staging in Ubiquitous High-end Homes. In CHI '12 Extended Abstracts on Human Factors in Computing Systems (CHI EA '12). Association for Computing Machinery, New York, NY, USA, 201210. https://doi.org/10.1145/2212776.2212798

[44] Nicolai Marquardt, Johannes Kiemer, and Saul Greenberg. 2010. What Caused That Touch?: Expressive Interaction with a Surface Through Fiduciary-tagged Gloves. In ACM International Conference on Interactive Tabletops and Surfaces (ITS '10). Association for Computing Machinery, New York, NY, USA, 139-142. https://doi.org/10.1145/1936652.1936680

[45] Matthew Mosher and David Tinapple. 2016. What We Have Lost / What We Have Gained: Tangible Interactions Between Physical and Digital Bodies. In Proceedings of the TEI '16: Tenth International Conference on Tangible, Embedded, and Embodied Interaction (TEI '16). Association for Computing Machinery, New York, NY, USA, 658-662. https://doi.org/10.1145/2839462.2856340

[46] Yohei Noguchi and Fumihide Tanaka. 2020. OMOY: A Handheld Robotic Gadget that Shifts its Weight to Express Emotions and Intentions. In Proceedings of the 2020 CHI Conference on Human Factors in Computing Systems (CHI '20). Association for Computing Machinery, New York, NY, USA, 1-13. DOI: https: //doi.org/10.1145/3313831.3376775

[47] Jifei Ou, Gershon Dublon, Chin-Yi Cheng, Felix Heibeck, Karl Willis, and Hiroshi Ishii. 2016. Cilllia: 3D Printed Micro-Pillar Structures for Surface Texture, Actuation and Sensing. In Proceedings of the $2016 \mathrm{CHI}$ Conference on Human Factors in Computing Systems (CHI '16). Association for Computing Machinery, New York, NY, USA, 5753-5764. DOI: https://doi.org/10.1145/2858036.2858257

[48] Amanda Parkes, Ivan Poupyrev, and Hiroshi Ishii. 2008. Designing Kinetic Interactions for Organic User Interfaces. Communications of the ACM 51, 6: 58-65. https://doi.org/10.1145/1349026.1349039

[49] Aman Parnami, Apurva Gupta, Gabriel Reyes, Ramik Sadana, Yang Li, and Gregory D. Abowd. 2016. Mogeste: A Mobile Tool for In-Situ Motion Gesture Design. In Proceedings of the 8th Indian Conference on Human Computer Interaction (IHCI '16). Association for Computing Machinery, New York, NY, USA, 35-43. https://doi.org/10.1145/3014362.3014365

[50] James Patten, Ben Recht, and Hiroshi Ishii. 2006. Interaction Techniques for $\mathrm{Mu}$ sical Performance with Tabletop Tangible Interfaces. In Proceedings of the 2006 ACM SIGCHI International Conference on Advances in Computer Entertainment Technology (ACE '06). Association for Computing Machinery, New York, NY, USA. https://doi.org/10.1145/1178823.1178856

[51] Marianne Graves Petersen, Ole Sejer Iversen, Peter Gall Krogh, and Martin Ludvigsen. 2004. Aesthetic Interaction: a pragmatist's aesthetics of interactive systems. In Proceedings of the 5th conference on Designing interactive systems: processes, practices, methods, and techniques. Association for Computing Machinery, New York, NY, USA 269-276. http://dl.acm.org/citation.cfm?id=1013153

[52] Stefano Piana, Paolo Alborno, Radoslaw Niewiadomski, Maurizio Mancini, Gualtiero Volpe, and Antonio Camurri. 2016. Movement Fluidity Analysis Based on Performance and Perception. In Proceedings of the 2016 CHI Conference Extended Abstracts on Human Factors in Computing Systems (CHI EA '16). Association for Computing Machinery, New York, NY, USA 1629-1636. https://doi.org/10.1145/2851581.2892478

[53] Ivan Poupyrev, Tatsushi Nashida, Shigeaki Maruyama, Jun Rekimoto, and Yasufumi Yamaji. 2004. Lumen: Interactive Visual and Shape Display for Calm Computing. In ACM SIGGRAPH 2004 Emerging Technologies (SIGGRAPH '04). Association for Computing Machinery, New York, NY, USA 17-. https: //doi.org/10.1145/1186155.1186173

[54] Majken K. Rasmussen, Esben W. Pedersen, Marianne G. Petersen, and Kasper Hornbæk. 2012. Shape-changing interfaces: a review of the design space and open research questions. In Proceedings of the SIGCHI Conference on Human 
Factors in Computing Systems (CHI '12). Association for Computing Machinery, New York, NY, USA, 735-744. DOI: https://doi.org/10.1145/2207676.2207781

[55] Hayes Solos Raffle, Amanda J. Parkes, and Hiroshi Ishii. 2004. Topobo: A Constructive Assembly System with Kinetic Memory. In Proceedings of the SIGCHI Conference on Human Factors in Computing Systems (CHI '04). Association for Computing Machinery, New York, NY, USA, 647-654. https://doi.org/10.1145/ 985692.985774

[56] Stuart Reeves, Steve Benford, Claire O'Malley, and Mike Fraser. 2005. Designing the Spectator Experience. In Proceedings of the SIGCHI Conference on Human Factors in Computing Systems (CHI '05). Association for Computing Machinery, New York, NY, USA, 741-750. https://doi.org/10.1145/1054972.1055074

[57] Vincent van Rheden and Bart Hengeveld. 2016. Engagement Through Embod iment: A Case For Mindful Interaction. In Proceedings of the TEI '16: Tenth International Conference on Tangible, Embedded, and Embodied Interaction (TEI '16). Association for Computing Machinery, New York, NY, USA, 349-356. https://doi.org/10.1145/2839462.2839498

[58] Shannon Rodgers, Bernd Ploderer, Brittany Maloney, and Jason Hang. 2019 Designing for Wellbeing-as-Interaction. In Extended Abstracts of the $2019 \mathrm{CHI}$ Conference on Human Factors in Computing Systems (CHI EA '19). Association for Computing Machinery, New York, NY, USA, Paper LBW1817, 1-6. DOI: https://doi.org/10.1145/3290607.3312901

[59] Philip R. Ross. 2008. Ethics and aesthetics in intelligent product and system design. Eindhoven University of Technology, Eindhoven, The Netherlands

[60] Philip R. Ross and David V. Keyson. 2007. The Case of Sculpting Atmospheres: Towards Design Principles for Expressive Tangible Interaction in Control of Ambient Systems. Personal Ubiquitous Computing 11, 2: 69-79. https://doi.org/ 10.1007/s00779-005-0062-3

[61] Philip R. Ross and Stephan A.G. Wensveen. 2010. Designing Behavior in Interaction: Using Aesthetic Experience as a Mechanism for Design. International Journal of Design 4, 2: 3-13.

[62] Beat Rossmy and Alexander Wiethoff. 2019. StringTouch: A Scalable Low-Cost Concept for Deformable Interfaces. In Extended Abstracts of the $2019 \mathrm{CHI}$ Conference on Human Factors in Computing Systems (CHI EA '19). Association for Computing Machinery, New York, NY, USA, Paper INT004, 1-4. DOI https://doi.org/10.1145/3290607.3313245

[63] Sichao Song and Seiji Yamada. 2018. Effect of Expressive Lights on Human Perception and Interpretation of Functional Robot. In Extended Abstracts of the 2018 CHI Conference on Human Factors in Computing Systems (CHI EA '18) Association for Computing Machinery, New York, NY, USA, Paper LBW629, 1-6. DOI: https://doi.org/10.1145/3170427.3188547

[64] Anna Ståhl, Petra Sundström, and Kristina Höök. 2005. A Foundation for Emotional Expressivity. In Proceedings of the 2005 Conference on Designing for User eXperience (DUX '05). Association for Computing Machinery, New York, NY, USA. http://dl.acm.org/citation.cfm?id=1138235.1138274

[65] Jelle Stienstra, Miguel Bruns Alonso, Stephan Wensveen, and Stoffel Kuenen. 2012 How to design for transformation of behavior through interactive materiality. In Proceedings of the 7th Nordic Conference on Human-Computer Interaction: Making Sense Through Design (NordiCHI '12). Association for Computing Machinery, New York, NY, USA, 21-30. DOI: https://doi.org/10.1145/2399016.2399020

[66] Rob Strong and Bill Gaver. 1996. Feather, scent and shaker: supporting simple intimacy. In Proceedings of CSCW, 29-30.

[67] Alex Taylor. 2015. After Interaction. Interactions 22, 5: 48-53. https://doi.org/10 $1145 / 2809888$

[68] Jonas Togler, Fabian Hemmert, and Reto Wettach. 2009. Living Interfaces: The Thrifty Faucet. In Proceedings of the 3rd International Conference on Tangible and Embedded Interaction (TEI '09). Association for Computing Machinery, New York, NY, USA, 43-44. https://doi.org/10.1145/1517664.1517680

[69] Theophanis Tsandilas, Catherine Letondal, and Wendy E. Mackay. 2009. Musink: Composing Music Through Augmented Drawing. In Proceedings of the SIGCHI Conference on Human Factors in Computing Systems (CHI '09). Association for Computing Machinery, New York, NY, USA, 819-828. https://doi.org/10.1145/ 1518701.1518827

[70] Anna Vallgårda. 2008. PLANKS: A Computational Composite. In Proceedings of the 5th Nordic Conference on Human-computer Interaction: Building Bridges (NordiCHI '08). Association for Computing Machinery, New York, NY, USA 569-574. https://doi.org/10.1145/1463160.1463248
[71] Anna Vallgårda. 2014. Giving Form to Computational Things: Developing a Practice of Interaction Design. Personal Ubiquitous Computing 18, 3: 577-592. https://doi.org/10.1007/s00779-013-0685-8

72] Anna Vallgårda and Tomas Sokoler. 2009. A Material Focus: Exploring Properties of Computational Composites. In CHI '09 Extended Abstracts on Human Factors in Computing Systems (CHI EA '09). Association for Computing Machinery, New York, NY, USA, 4147-4152. https://doi.org/10.1145/1520340.1520631

[73] Gil Weinberg and Seum-Lim Gan. 2001. The Squeezables: Toward an Expressive and Interdependent Multi-player Musical Instrument. Computer Music Journal 25, 2: 37-45. https://doi.org/10.1162/014892601750302570

[74] Anna Weisling. 2017. The Distaff: A Physical Interface to Facilitate Interdisciplinary Collaborative Performance. In Proceedings of the 2017 Conference on Designing Interactive Systems (DIS '17). Association for Computing Machinery, New York, NY, USA, 1365-1368. https://doi.org/10.1145/3064663.3064713

[75] Stephan Wensveen, Tom Djajadiningrat, and Kees Overbeeke. 2004. Interaction Frogger: A Design Framework to Couple Action and Function Through Feedback and Feedforward. In Proceedings of the 5th Conference on Designing Interactive Systems: Processes, Practices, Methods, and Techniques (DIS '04). Association for Computing Machinery, New York, NY, USA, 177-184. https://doi.org/10.1145/ 1013115.1013140

[76] Stephan Wensveen, Kees Overbeeke, and Tom Djajadiningrat. 2002. Push Me, Shove Me and I Show You How You Feel: Recognising Mood from Emotionally Rich Interaction. In Proceedings of the 4th Conference on Designing Interactive Systems: Processes, Practices, Methods, and Techniques (DIS '02). Association for Computing Machinery, New York, NY, USA, 335-340. https://doi.org/10.1145/ 778712.778759

[77] Stephen Wensveen, Kees Overbeeke, and Tom Djajadiningrat. 2000. Touch Me, Hit Me and I Know How You Feel: A Design Approach to Emotionally Rich Interaction. In Proceedings of the 3rd Conference on Designing Interactive Systems: Processes, Practices, Methods, and Techniques (DIS '00). Association for Computing Machinery, New York, NY, USA, 48-52. https://doi.org/10.1145/347642.347661

[78] Gerard Wilkinson, Ahmed Kharrufa, Jonathan Hook, Bradley Pursglove, Gavin Wood, Hendrik Haeuser, Nils Y. Hammerla, Steve Hodges, and Patrick Olivier. 2016. Expressy: Using a Wrist-worn Inertial Measurement Unit to Add Expressiveness to Touch- based Interactions. In Proceedings of the 2016 CHI Conference on Human Factors in Computing Systems (CHI '16). Association for Computing Machinery, New York, NY, USA, 2832-2844. DOI: https://doi.org/10.1145/2858036. 2858223

[79] Cara Wilson, Laurianne Sitbon, Bernd Ploderer, Jeremy Opie, and Margot Brereton. 2020. Self-Expression by Design: Co-Designing the ExpressiBall with Minimally-Verbal Children on the Autism Spectrum. In Proceedings of the 2020 CHI Conference on Human Factors in Computing Systems (CHI '20). Association for Computing Machinery, New York, NY, USA, 1-13. DOI: https: //doi.org/10.1145/3313831.3376171

[80] Haijun Xia, Ken Hinckley, Michel Pahud, Xiao Tu, and Bill Buxton. 2017. WritLarge: Ink Unleashed by Unified Scope, Action, \& Zoom. In Proceedings of the 2017 CHI Conference on Human Factors in Computing Systems (CHI '17). Association for Computing Machinery, New York, NY, USA, 3227-3240. https://doi.org/10.1145/3025453.3025664

[81] Yuhui You, Mitchell Fogelson, Kelvin Cheng, and Bjorn Stenger. 2020. EMI: An Expressive Mobile Interactive Robot. In Extended Abstracts of the $2020 \mathrm{CH}$ Conference on Human Factors in Computing Systems (CHI EA '20). Association for Computing Machinery, New York, NY, USA, 1-8. DOI: https://doi.org/10.1145/ 3334480.3382852

[82] Malgorzata A. Zboinska, Delia Dumitrescu, and Hanna Landin. 2019. Expressing and Sensing Hybrid Materiality: Voluminous Interactive Architectural Substance. In Proceedings of the Thirteenth International Conference on Tangible, Embedded, and Embodied Interaction (TEI '19). Association for Computing Machinery, New York, NY, USA, 483-489. DOI: https://doi.org/10.1145/3294109.3301265

[83] Jan Zekveld, Mathias Funk, and Saskia Bakker. 2016. The Tumble Clock: Bringing Users in Touch with Their Snooze Time. In Proceedings of the 2016 ACM Conference on Designing Interactive Systems (DIS '16). Association for Computing Machinery, New York, NY, USA, 900-904. https://doi.org/10.1145/2901790.2901857

[84] Yuhang Zhao, Shaomei Wu, Lindsay Reynolds, and Shiri Azenkot. 2018. A Face Recognition Application for People with Visual Impairments: Understanding Use Beyond the Lab. In Proceedings of the 2018 CHI Conference on Human Factors in Computing Systems (CHI '18). Association for Computing Machinery, New York, NY, USA, Paper 215, 1-14. DOI: https://doi.org/10.1145/3173574.3173789 